\title{
Knowledge and practices regarding standard precautions among supportive staff in the National Hospital of Sri Lanka
}

\author{
A. D. Harshana ${ }^{* 1}$, R. G. Y. Hansini ${ }^{* 1}$, S. G. L. S. Hansini ${ }^{* 1}$, Y. Mathangasinghe ${ }^{2}$ \\ ${ }^{1}$ Faculty of Medicine, University of Colombo, Sri Lanka \\ ${ }^{2}$ Department of Anatomy, Faculty of Medicine, University of Colombo, Sri Lanka \\ *Co-first authors (equal contributions)
}

Keywords: Standard precautions; hospital acquired infections; health care workers; knowledge; practices

\begin{abstract}
\section{Introduction}

Adherence to standard precautions significantly reduces the transmission of hospital-acquired infections. We intended to assess the knowledge and practices regarding standard precautions among supportive staff members in the National Hospital of Sri Lanka (NHSL).
\end{abstract}

\section{Methods}

A descriptive cross-sectional study was conducted among supportive staff members at NHSL. They were selected using stratified one-stage cluster sampling and their knowledge and practices regarding standard precautions were assessed using an interviewer-administrated questionnaire.

\section{Results}

Of 108 participants, 69 (63.9\%) were aged 20-40 years. Onehundred and five (97.2\%) were educated up to Ordinary Level and $48(44.4 \%)$ had working experience of more than six years. Twenty-six had at least one needle-stick injury during their career. Six $(5.6 \%)$ have not been vaccinated for Hepatitis $\mathrm{B}$ since their recruitment. Mean knowledge and practice scores were $66.3 \pm 12.5 \%$ and $70.0 \pm 7.7 \%$ respectively. There were no differences in knowledge scores based on the level of education $(\mathrm{t}=1.19, \mathrm{p}=0.24)$ and the total years of service $(\mathrm{t}=$ $0.44, p=0.66$ ). Similarly, there were no differences in practising proper precautions based on their educational level $(\mathrm{t}=0.25, \mathrm{p}=0.80)$ or the duration of service $(\mathrm{t}=0.87, \mathrm{p}=0.39)$. However, the knowledge score positively correlated with the good practice score $(r=0.197, \mathrm{p}=0.04)$.

\section{Conclusions}

The supportive staff members with good knowledge were more adherent to the correct practice. Neither the level of education nor the experience was associated with their knowledge or good practices. Provided the potential risk of

\footnotetext{
Correspondence: Yasith Mathangasinghe

E-mail: yasith@anat.cmb.ac.lk

(iDhttps://orcid.org/0000-0003-4641-5642

Received: 08-01-2020 Accepted: 15-06-2020

DOI: http://doi.org/10.4038/sljs.v38i2.8663
}

transmission of blood-borne infections, Hepatitis B vaccination and post-vaccination antibody testing could be made mandatory before starting their carrier.

\section{Introduction}

Hospital Acquired Infections (HAI), a.k.a. Healthcare Associated Infections are a major public health burden and it is estimated to account for the annual expenditure of $\$ 6.5$ billion in the USA [1] while contributing to significant morbidity and mortality [2,3]. One of the most cost-effective strategies to prevent HAIs is adhering to a set of guidelines referred to as standard precautions which minimize the spread of infective organisms from one person to another [4].

The World Health Organization defines the standard precautions as infection control safety measures to minimize the transmission of infectious microorganisms from known and unknown sources [5]. Studies have shown that the adherence to these guidelines by the health care workers is not optimum [6,7], thus increasing the transmission of diseases at health care settings, both to the patients and the healthcare workers [8]. The supportive healthcare staff a.k.a. hospital attendants play a major role in transporting blood and body fluids, maintaining the hygiene of patients and ensuring the cleanliness of the ward. Nonetheless, the role of the supportive healthcare staff in adhering to standard precautions are explored sparsely in the literature, especially in the developing countries like Sri Lanka where patient load per health care worker is comparatively high [9]. Therefore, the objective of this study was to assess knowledge, practices and associated factors for the adherence to the standard precautions among supportive staff in the National Hospital of Sri Lanka (NHSL).

\section{Methods}

A descriptive cross-sectional study was conducted among the supportive healthcare workers (attendants) of the NHSL from February to May 2019. Those who have at least six months of work experience in the patient handling units of the hospital were recruited for the study. A stratified one-stage cluster sampling method was used to obtain a representative sample from the entire hospital. The supportive staff of the NHSL is categorized into 13 sections for administrative purposes. Usually, more than 100 support staff members are working 
under each section. Of them, two sections (kitchen and officers' sections) are not involved in patient handling, thus were excluded from the study. Of the remaining 11 strata, 27 clusters were randomly identified based on the wards/units where they work, and the final sample size was 108 . The details of sampling and the sizes of clusters in each stratum are summarized in the Electronic Supplementary Material 1.

An interviewer-administered questionnaire was used for the data collection. The questionnaire was designed to collect sociodemographic data, knowledge and practices on standard precautions on day-to-day patient care. The knowledge was assessed using true/false and single-best-response type questions and was weighted according to the importance of the precaution or the technique predetermined by experts. The practices were measured on 10-point Likert scales.

Cumulative scores were subsequently computed for both knowledge and practices, and the total scores thus computed were 100 marks each for knowledge and practice. Betweengroup comparisons of continuous data were conducted using t-tests while associations between two continuous variables were explored using Pearson's correlation coefficient test. All the statistical analyses were conducted using Statistical Package for Social Sciences (SPSS) version 26 and GraphPad Prism version 8.4.2 at a significance level of 0.05 . The study protocol was approved by the Ethics Review Committees of the Faculty of Medicine, University of Colombo (CSRP/19/018) and the National Hospital of Sri Lanka (ETH/COM/2018/13).

\section{Results}

Of 108 eligible supportive staff members invited for the study, the positive response rate was $100 \%$. The sociodemographic characteristics of the study sample are summarized in Figure1.

Out of seven questions regarding basic knowledge on standard precautions, most of the participants $(88.9 \%, \mathrm{n}=96)$ answered correctly to the question regarding the importance of handwashing after touching blood or body fluid, even if gloves were worn. Only $35.2 \%(n=38)$ answered correctly to the question that all patients should be considered as infected with Human Immunodeficiency Virus (HIV) unless proven otherwise, which had the worst proportion of correct responses among the questions. Fifty-seven participants $(52.8 \%)$ correctly stated that the base of the thumb was the most vulnerable site of germ accumulation in hand. The necessity of handwashing before touching the patients, when touching one patient after touching another, before handling invasive devices and when changing the procedures of the same patient if there is contamination with body fluids, was known by 97 (89.8\%), 104 (96.3\%), 85 (78.7\%) and 67
$(62.0 \%)$ participants respectively. Majority of participants $(64.8 \%, n=70)$ did not know that they must wash their eyes for at least 15 minutes in case of an eye splash with body fluids. However, the colour code of disposal of contaminated material was correctly answered by the majority $(96.3 \%$, $\mathrm{n}=104)$.

Distribution of practices regarding standard precautions on different health care practices of the supportive staff is shown in Figure 2. Interestingly, only $58.3 \%(n=63)$ of the staff members routinely practised washing hands before touching patients. Most of the participants washed their hands only for 10 to 20 seconds $(31.5 \%, n=34)$ while only nine $(8.3 \%)$ spent more than forty seconds for hand washing. Most of them $(n=93,86.1 \%)$ preferred soap and water when washing hands. Sixty-three participants $(61.7 \%)$ declared that they routinely recapped the contaminated needles and 26 participants (24.1\%) had experienced needlestick injuries in their carrier. Of those who had needle stick injuries, twenty (76.9\%) had sought further medical management after the incident while three participants $(11.5 \%)$ had ignored the incident. Six (5.6\%) participants had never received the hepatitis B vaccination since recruitment. Only 98 participants (90.7\%) had received a formal learning session on standard precautions during their carrier. Mean knowledge and practice scores were $66.3 \pm 12.5 \%$ and $70.0 \pm 7.7 \%$ respectively. There were no significant differences in knowledge scores based on the level of education (i.e. below and above advanced level $)(\mathrm{t}=1.19, \mathrm{p}=0.24)$ and the total years of service (i.e. below and above six years) $(\mathrm{t}=0.44, \mathrm{p}=0.66)$. Similarly, there were no difference in practising proper precautions based on their educational level $(t=0.25, \mathrm{p}=0.80)$ or the duration of service $(\mathrm{t}=0.87, \mathrm{p}=0.39)$. However, the knowledge score positively correlated with the good practice $\operatorname{score}(r=0.197, p=0.04)$.

\section{Discussion}

In our study, we identified several knowledge gaps among the supportive healthcare staff members. Over $10 \%$ of the study sample was unaware of the fact that handwashing needs to be practised before and after touching a patient, while more than one-third of the sample was ignorant of the importance of handwashing in between procedures conducted on the same patient, particularly if there is contamination with the body fluids of the patient. Regardless of the knowledge of proper handwashing practices, the proportion of those who practised handwashing before touching a patient was 58\%. Intriguingly, this figure highlights the importance of implementing regulations and conducting routine audits to ensure the adherence to the standard precautions, since only providing with knowledge might not be adequate to motivate the healthcare workers to practice them like in the previous reports [6]. 
A)

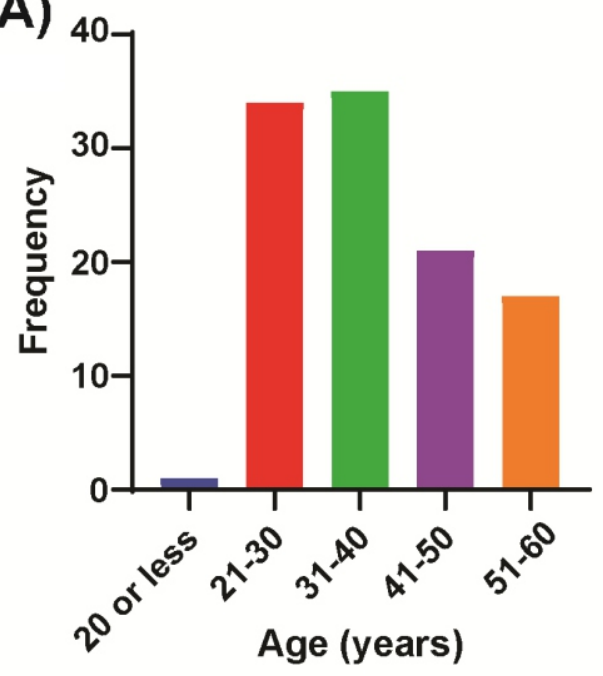

C)

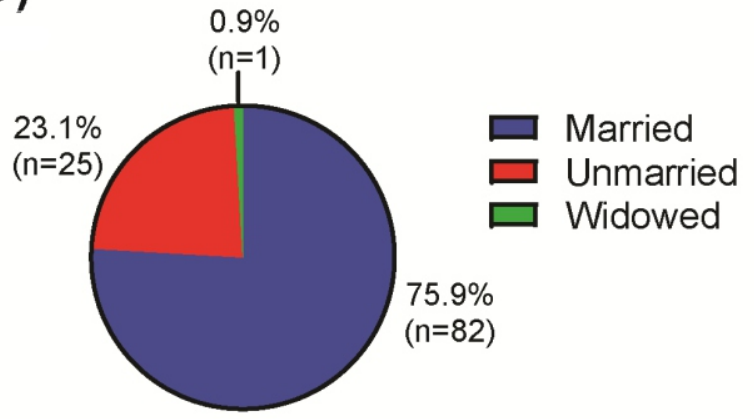

E)

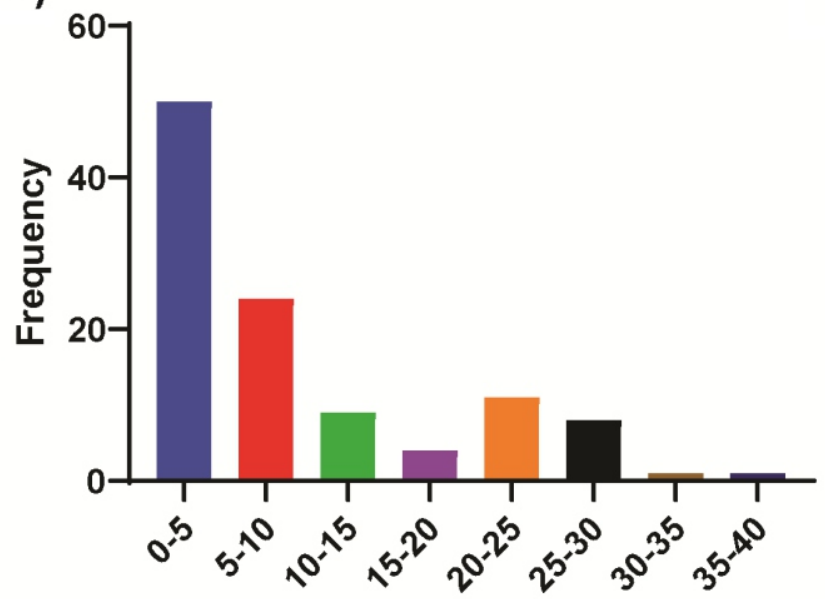

B)

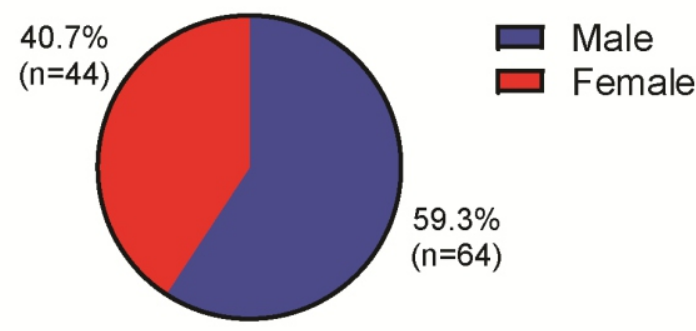

D)
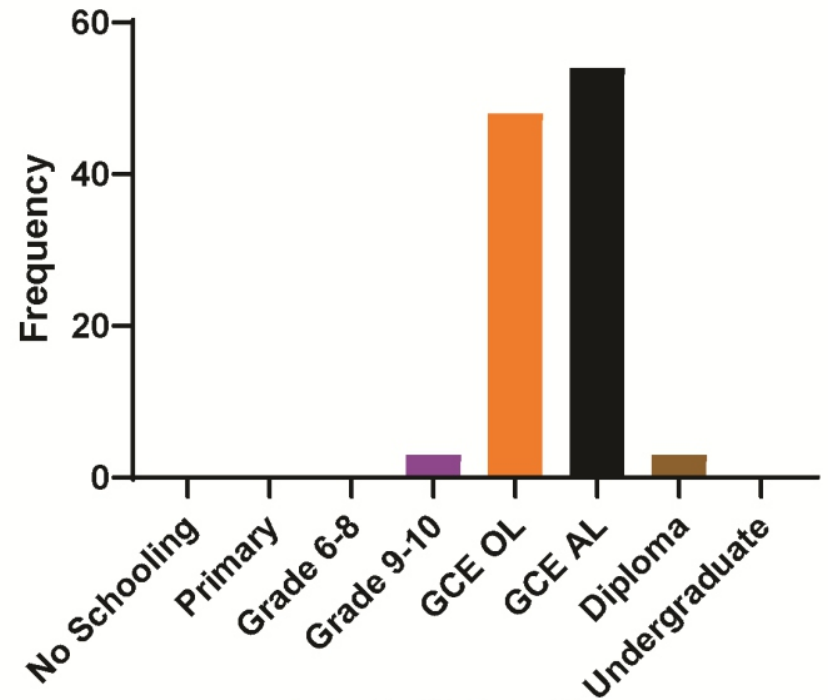

Level of Education

F)

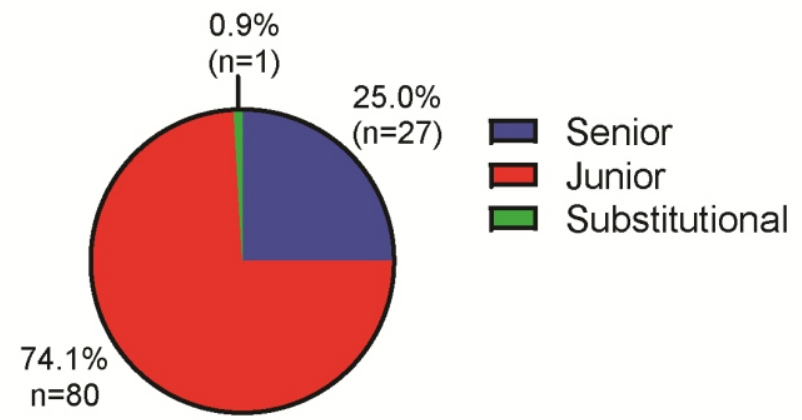

Figure 1. Sociodemographic characteristics of the study sample $\mid$ A. Distribution of the age B. Distribution of the sex C. Distribution of the marital status D. Distribution of the highest completed educational level E. Distribution of the total duration of service as a supportive healthcare worker F. Distribution of the designation. Abbreviations: GCE - General Certificate of Education; O/L - Ordinary Level; A/L - Advanced Level 
A)

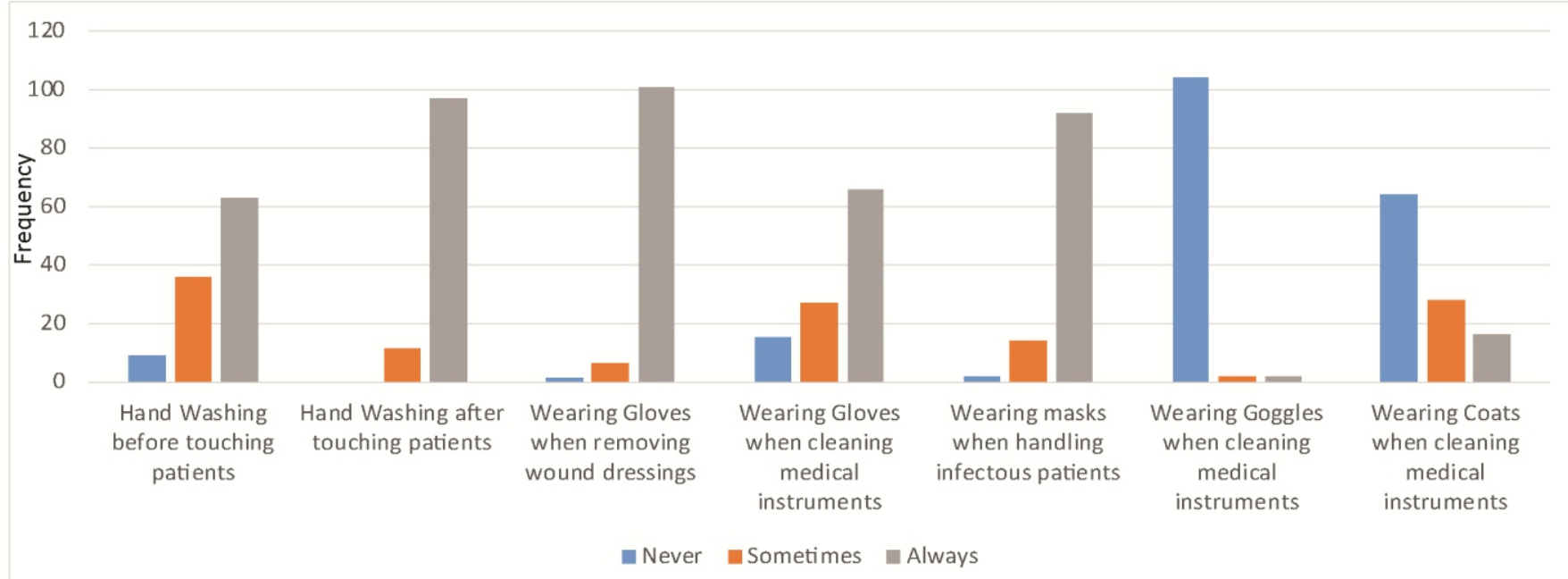

\section{B)}
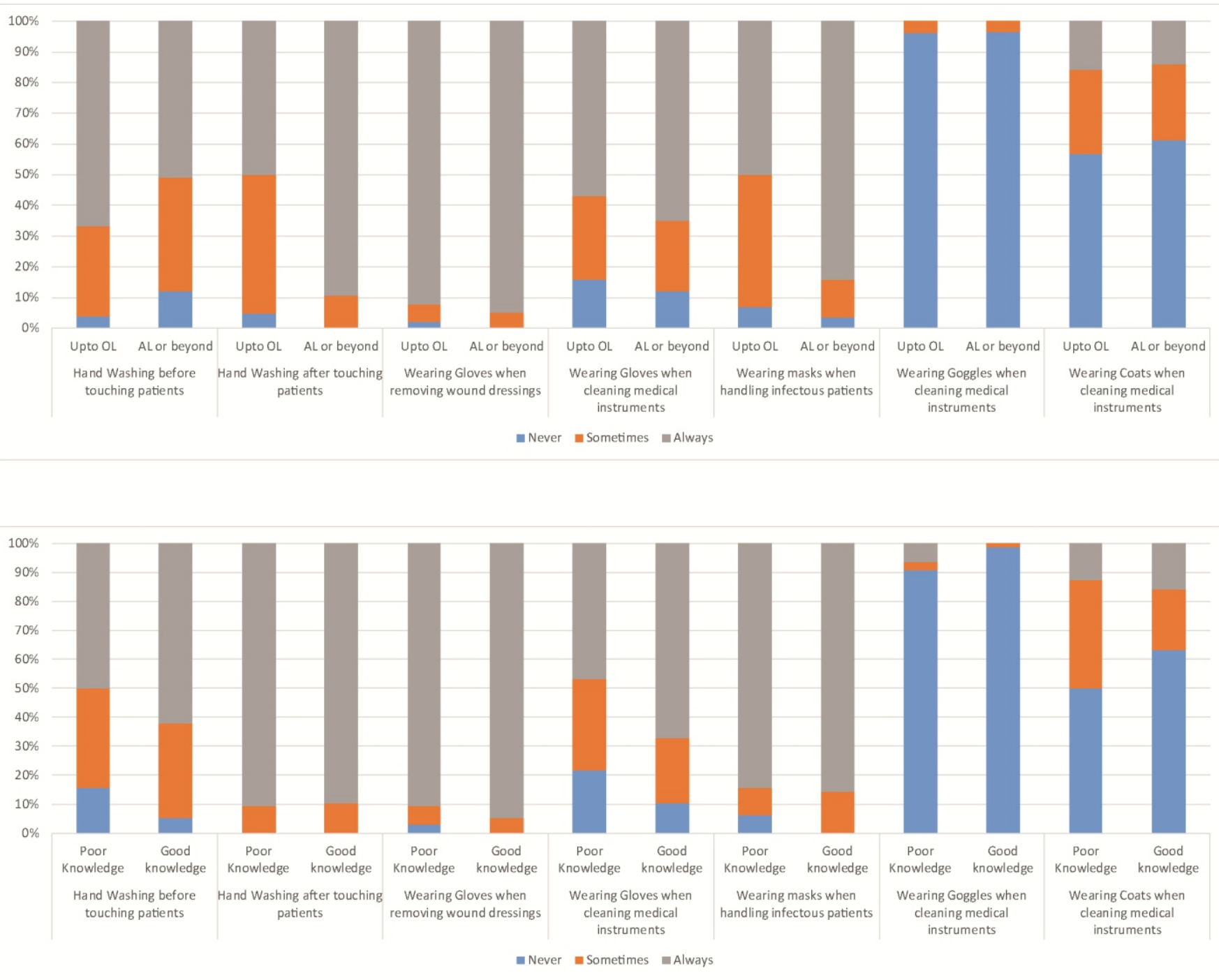

Figure 2. Distribution of practices regarding standard precautions of different health care practices of supportive staff $\mid$ A. A composite bar chart shows the standard precautions practiced by the participants. Since questions on practices are answered on $10-$ point Likert scale, the three categories are determined as follows: $1=$ never, $2-9=$ sometimes, $10=$ always B. Percentage component bar charts illustrate the practices according to the highest completed level of education (top) and the level of knowledge (bottom). The level of knowledge is categorized into two groups; poor knowledge and good knowledge; by splitting the knowledge score about the median. Abbreviations: GCE - General Certificate of Education; O/L - Ordinary Level; A/L - Advanced Level 
Body fluid splashes on eyes not only cause blindness but also act as a source for the transmission of infections [10, 11]. According to a prospective national study conducted in France, healthcare workers were frequently affected by eye splashes with blood or body fluids, of which $39 \%$ cases were preventable if proper standard precautions were followed [12]. The most effective first aid measures to be taken irrespective of the splash is to wash eyes with running water for at least 15 minutes [13]. Nonetheless, this correct first aid measures for eye splashes with body fluids was not known by approximately two-thirds of the supportive healthcare workers, whose knowledge was comparatively poor than the first-year medical students [14] in a Sri Lankan Medical Faculty.

Even though venepuncture is routinely conducted by the nurses, the supportive healthcare workers are also exposed to contaminated syringes and needles in routine ward and theatre work. Regrettably, recapping of the used needles was practised by $61.7 \%$ of the study sample, which is higher than the reported proportions of recapping practised by the nurses in several previous studies $[15,16]$.

Inadequacy of the knowledge that all the patients should be considered as infected with HIV unless proven otherwise may be one of the reasons for practising routine recapping of the used needles and the inadequate use of gloves by the supportive healthcare workers, which the authors have observed in the state sector hospitals. Moreover, poor knowledge may have contributed to ignoring needle stick injuries by some of the staff members. This needs the urgent attention of the medical administrators and strategies such as periodic awareness programmes and post-exposure prophylaxis protocols need to be implemented. The importance of such periodic training programmes is further highlighted by the fact that approximately $10 \%$ of our study sample being never taught regarding standard precautions during their career and six participants had not being vaccinated for Hepatitis B since recruitment.

We found that those who had good knowledge scores adhered to the standard precautions well, however, their education level or the experience did not significantly associate with good practice. Similar to our study, the education level had no association with the adherence to the standard precautions among radiographers in a tertiary care hospital of Sri Lanka [17]. However, a Sri Lankan study conducted among the nursing staff at the same setting concluded that the junior nurses were more compliant on the standard precautions than the senior nursing staff, probably since the former have updated their knowledge recently [18].
Hence, we recommend organizing awareness programmes targeting supportive staff, both new recruits and experienced staff members, which might be helpful in reducing hospitalacquired infections in the long run.

\section{Limitations}

In this study, we assessed the practices by questioning the individuals rather than directly observing. Furthermore, since we used interviewer-administered questionnaires, the responses towards practices might have been biased since participants may not have disclosed their bad practices to the interviewers. The sample size of 108 was employed based on the availability of logistics for the study rather than using an objective calculation.

\section{Conclusions}

There were several gaps regarding the indications for handwashing, first aid measures in case of an eye splash and recapping of the contaminated needles among the supportive healthcare workers in our study sample. The supportive staff members with a good knowledge of standard precautions were more adherent to the correct practice. Neither the level of education nor the experience associated with their knowledge or good practices. Moreover, provided the potential risk of transmission of blood-borne infections, Hepatitis B vaccination and post-vaccination antibody testing could be made mandatory before starting their carrier.

\section{Acknowledgement}

This research was carried out as a requirement of the Community Stream undergraduate academic programme of the Faculty of Medicine, University of Colombo, Sri Lanka.

All authors disclose no conflict of interest. The study was conducted in accordance with the ethical standards of the relevant institutional or national ethics committee and the Helsinki Declaration of 1975, as revised in 2000.

\section{References}

1. Scott, R.D., The direct medical costs of healthcare-associated infections in US hospitals and the benefits of prevention. 2009.

2. Kwon, J.H., M.A. Olsen, and E.R. Dubberke, The morbidity, mortality, and costs associated with Clostridium difficile infection. Infectious disease clinics of North America, 2015. 29(1): p. 123-134.

https://doi.org/10.1016/j.idc.2014.11.003

3. Cornejo-Juárez, P., D. Vilar-Compte, C. Pérez-Jiménez, et al., The impact of hospital-acquired infections with multidrug-resistant bacteria in an oncology intensive care unit. International Journal of Infectious Diseases, 2015. 31: p. 31-34.

https://doi.org/10.1016/j.ijid.2014.12.022 
4. Arefian, H., M. Vogel, A. Kwetkat, and M. Hartmann, Economic evaluation of interventions for prevention of hospital acquired infections: a systematic review. PLoS One, 2016. 11(1): p. e0146381.https://doi.org/10.1371/journal.pone.0146381

5. World Health Organization. Standard Precautions on Healthcare. 2007 [Accessed on 07/01/2020]; Available from: https://www.who.int/docs/default-source/documents/healthtopics/standard-precautions-in-health- care.pdf?sfvrsn $=7 \mathrm{c} 453$ df0_2.

6. Ogoina, D., K. Pondei, B. Adetunji, et al., Knowledge, attitude and practice of standard precautions of infection control by hospital workers in two tertiary hospitals in Nigeria. Journal of infection prevention, 2015. 16(1): p. 16-22. https://doi.org/10.1177/1757177414558957

7. Haile, T.G., E.H. Engeda, and A.A. Abdo, Compliance with standard precautions and associated factors among healthcare workers in Gondar University Comprehensive Specialized Hospital, Northwest Ethiopia. Journal of environmental and public health, 2017.2017.

https://doi.org/10.1155/2017/2050635

8. Sridhar, M., S. Boopathi, R. Lodha, and S. Kabra, Standard precautions and post exposure prophylaxis for preventing infections. The Indian Journal of Pediatrics, 2004. 71(7): p. $617-$ 625. https://doi.org/10.1007/BF02724121

9. Russell, S., Treatment-seeking behaviour in urban Sri Lanka: trusting the state, trusting private providers. Social science \& medicine, 2005. 61(7): p. 1396-1407.

https://doi.org/10.1016/j.socscimed.2004.11.077

10.Siegel, J.D., E. Rhinehart, M. Jackson, and L. Chiarello, 2007 Guideline for isolation precautions preventing transmission of infectious agents in healthcare settings. 2007.

https://doi.org/10.1016/j.ajic.2007.10.007

11.Davies, C., M. Khan, A. Ghauri, and C. Ranaboldo, Blood and body fluid splashes during surgery-the need for eye protection and masks. The Annals of The Royal College of Surgeons of England, 2007. 89(8): p. 770-772.

https://doi.org/10.1308/003588407X209301
12.Venier, A., A. Vincent, F. L'heriteau, et al., Surveillance of occupational blood and body fluid exposures among French healthcare workers in 2004. Infection Control \& Hospital Epidemiology, 2007.28(10):p. 1196-1201.

https://doi.org/10.1086/520742

13.Mayo Clinic. Chemical Splash in the Eye. 2018 [Accessed on 07/01/2020]; Available from:

https://www.mayoclinic.org/first-aid/first-aid-eyeemergency/basics/art-20056647.

14.Mathangasinghe, Y., U. Samaranayake, M. Perera, et al., An audit on medical students' exposures to occupational hazards during cadaveric dissections. Sri Lanka Anatomy Journal, 2019. 3(1). https://doi.org/10.4038/slaj.v3i1.50

15.Nsubuga, F.M. and M.S. Jaakkola, Needle stick injuries among nurses in sub-Saharan Africa. Tropical medicine \& international health, 2005. 10(8): p. 773-781.

https://doi.org/10.1111/j.1365-3156.2005.01453.x

16.Galougahi, M.H.K., Evaluation of needle stick injuries among nurses of Khanevadeh Hospital in Tehran. Iranian journal of nursing and midwifery research, 2010. 15(4): p. 172.

17. Jayasinghe, R.D. and B.S. Weerakoon, Prevention of nosocomial infections and standard precautions: knowledge and practice among radiographers in Sri Lanka. Journal of Medical \& Allied Sciences, 2014.4(1): p. 9 . https://doi.org/10.4038/slja.v18i2.2446

18. Bataduwaarachchi, V., M. Balasubramanium, D. Balasooriya, and L. Senerath, Compliance with aseptic precautions during intravenous access among the nursing staff at the National Hospital of Sri Lanka (NHSL). Int J Infect Control, 2011. 7(3). https://doi.org/10.3396/ijic.V7i3.021.11 